\title{
Results of the NaCo Large Program: probing the occurrence of exoplanets and brown dwarfs at wide orbit
}

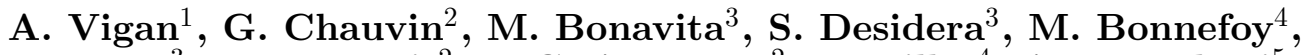
D. Mesa ${ }^{3}$, J.-L. Beuzit ${ }^{2}$, J.-C. Augereau ${ }^{2}$, B. Biller ${ }^{4}$, A. Boccaletti ${ }^{5}$, E. Brugaletta ${ }^{6}$, E. Buenzli ${ }^{7}$, J. Carson ${ }^{8}$, E. Covino ${ }^{9}$, P. Delorme ${ }^{2}$, A. Eggenberger ${ }^{2}$, M. Feldt ${ }^{4}$, J. Hagelberg ${ }^{10}$, T. Henning ${ }^{4}$, A.-M.

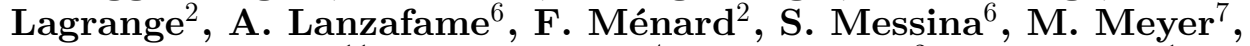
G. Montagnier ${ }^{11}$, C. Mordasini ${ }^{4}$, D. Mouillet ${ }^{2}$, C. Moutou ${ }^{1}$, L. Mugnier ${ }^{12}$, S. P. Quanz ${ }^{7}$, M. Reggiani ${ }^{7}$, D. Ségransan ${ }^{10}$, C. Thalmann ${ }^{13}$, R. Waters ${ }^{13}$ and A. Zurlo ${ }^{1}$

${ }^{1}$ Aix Marseille Université, CNRS, LAM (Laboratoire d'Astrophysique de Marseille) UMR 7326, 13388, Marseille, France. email: arthur.vigan@oamp.fr;

${ }^{2}$ UJF-Grenoble 1 / CNRS-INSU, IPAG, UMR 5274, Grenoble, F-38041, France;

${ }^{3}$ INAF - Osservatorio Astronomico di Padova, Vicolo dell' Osservatorio 5, 35122, Padova, Italy;

${ }^{4}$ Max Planck Instiute für Astronomy, Königsthul 17, D-69117 Heidelberg, Germany;

${ }^{5}$ LESIA, Observatoire de Paris, CNRS, University Pierre et Marie Curie Paris 6 and University Denis Diderot Paris 7, 5 place Jules Janssen, 92195 Meudon, France;

${ }^{6}$ INAF - Osservatorio Astrofisico di Catania, via S. Sofia 78, 95123 Catania, Italy;

${ }^{7}$ Institute for Astronomy, ETH Zurich, Wolfgang-Pauli-Strasse 27, 8093 Zurich, Switzerland;

${ }^{8}$ College of Charleston, Department of Physics \& Astronomy, USA;

${ }^{9}$ INAF - Osservatorio Astronomico di Napoli, Salita Moiariello 16, 80131 Napoli, Italy;

${ }^{10}$ Geneva Observatory, Univ. of Geneva, Chemin des Mailettes 51, 1290, Versoix, Switzerland;

${ }^{11}$ Observatoire de Haute-Provence, CNRS/OAMP, 04870 Saint-Michel-l'Observatoire, France;

${ }^{12}$ Onera - The French Aerospace Lab, 92322, Chatillon, France;

${ }^{13}$ University of Amsterdam, Postbus 94249, 1090 GE, Amsterdam, The Netherlands

\begin{abstract}
Over the past decade, a growing number of deep imaging surveys have started to provide meaningful constraints on the population of extrasolar giant planets at large orbital separation. Primary targets for these surveys have been carefully selected based on their age, distance and spectral type, and often on their membership to young nearby associations where all stars share common kinematics, photometric and spectroscopic properties. The next step is a wider statistical analysis of the frequency and properties of low mass companions as a function of stellar mass and orbital separation. In late 2009, we initiated a coordinated European Large Program using angular differential imaging in the $\mathrm{H}$ band $(1.66 \mu \mathrm{m})$ with $\mathrm{NaCo}$ at the VLT. Our aim is to provide a comprehensive and statistically significant study of the occurrence of extrasolar giant planets and brown dwarfs at large (5-500 AU) orbital separation around 150 young, nearby stars, a large fraction of which have never been observed at very deep contrast. The survey has now been completed and we present the data analysis and detection limits for the observed sample, for which we reach the planetary-mass domain at separations of $\gtrsim 50 \mathrm{AU}$ on average. We also present the results of the statistical analysis that has been performed over the 75 targets newly observed at high-contrast. We discuss the details of the statistical analysis and the physical constraints that our survey provides for the frequency and formation scenario of planetary mass companions at large separation.
\end{abstract}

Keywords. instrumentation: adaptive optics, instrumentation: high angular resolution, stars: imaging, methods: statistical 

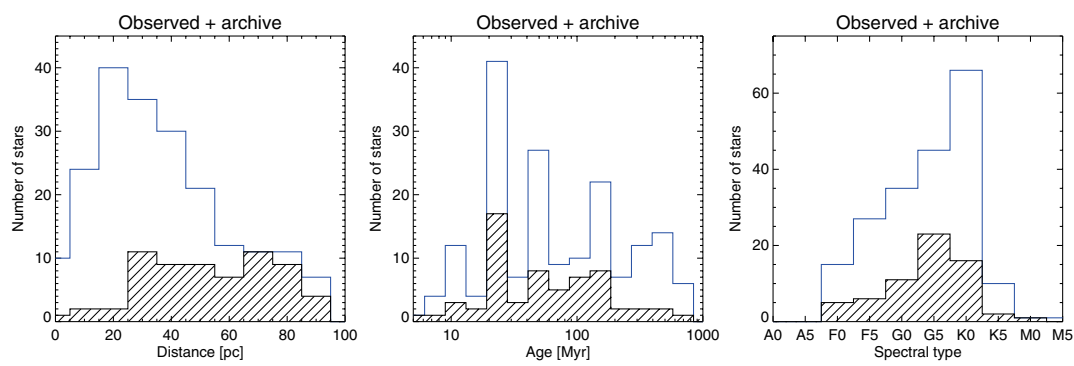

Figure 1. Distribution of NaCo-LP target sample in distance (left), age (center) and spectral type (right). The observed sample is plotted as the hatched black histogram, and the full sample for the statistical analysis as the blue histogram.

\section{Introduction}

The NaCo Large Program (NaCo-LP) project was initiated in 2008 as a large-scale European effort to measure the occurrence of giant exoplanets and brown dwarf at wide orbits (50-500 AU). This survey was also designed to prepare the near-infrared survey (NIRSUR) to be conducted with the SPHERE instrument (Beuzit et al. 2008). The $\mathrm{NaCo}-\mathrm{LP}$ would provide useful information such as first epoch observation for background contaminants and false alarm probability estimation. A total of 18 nights was allocated on $\mathrm{NaCo}$ at the VLT $\dagger$. We are now concluding the survey.

\section{Sample selection}

The target selection for the NaCo-LP was initially done within the SPHERE NIRSUR database. The following criteria were used: $\delta \leqslant 25^{\circ}, \mathrm{d} \leqslant 100 \mathrm{pc}$, age $\leqslant 200 \mathrm{Myr}, \mathrm{R} \leqslant$ $9.5 \mathrm{mag}, 0.4 \mathrm{mag} \leqslant B-V \leqslant 1.2 \mathrm{mag}$, no visual binaries within 6 ", no spectroscopic binaries. The $R$ magnitude threshold was designed to ensure that the targets observed in the NaCo-LP will also be observable in the future with the SPHERE instrument. The $B-V$ cut in color was designed to select only solar-type stars. This selection resulted in an initial sample of $\sim 200$ stars, which was then cross-correlated with previous and on-going surveys at the time (e.g. Masciadri et al. (2005); Biller et al. (2007); Lafrenière et al. (2007a); Chauvin et al. (2010), ...) in order to select only stars that had never been observed at high-contrast. The second selection step resulted in a sample of 110 stars. Both samples are illustrated in Fig. 1.

\section{Observations and data analysis}

The strategy was to observe in $H$-band with the $\mathrm{S} 13$ objective (13 mas/pixel, FoV 14 " $\times 14$ "), pupil-tracking mode for proper implementation of angular differential imaging (Marois et al. 2006), and the 0.7" Lyot coronagraph. Each observations consisted of a reference PSF (without coronagraph) obtained with a neutral density filter, followed by 45 minutes of deep coronagraphic images. Unfortunately, after this first observing run in December 2009, we discovered a problem with the pupil-tracking mode that induced a slow drift of the star behind the coronagraph that could reach several pixels per minutes. For the subsequent observing runs and follow-ups, the observing strategy was modified to saturated imaging with shorter integration times and a shift-and-addprocedure.

$\dagger$ ESO program 184.C-0567, PI J.-L. Beuzit, "Probing the Occurrence of Exoplanets and Brown Dwarfs at Wide Orbits". 

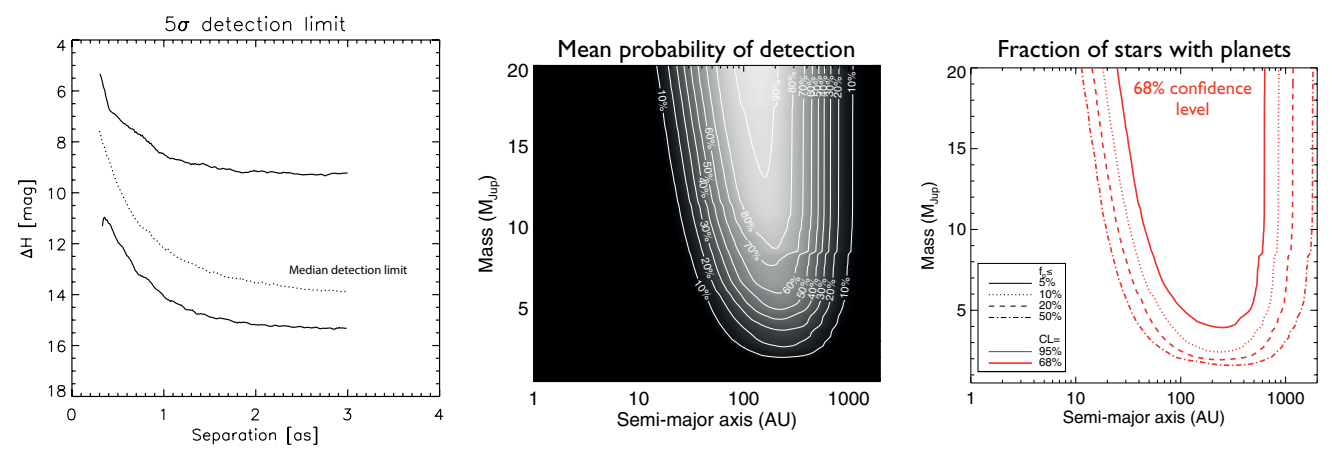

Figure 2. Left: Best (top), worst (bottom) and median (middle) $5 \sigma$ detection limits of the survey within 3 " given as the contrast with respect to the star in $H$-band. Center: mean probability of detection of the observed sample as a function of mass and semi-major axis. Right: Upper limit to the fraction of stars that can harbor a substellar companion, as a function of mass and semi-major axis, at a $68 \%$ confidence level, assuming a null-result for the survey.

The data analysis was performed using four independent pipelines, and two of them were used to reduce and analyze the complete survey (see descriptions in Vigan et al. 2012 and Rameau et al. 2013). The general scheme of the analysis consisted in five main steps: cleaning of the data, frame centering, bad frames rejection, ADI analysis, and astrometry and photometry of the candidates. The results presented here are based on an analysis using LOCI (Lafrenière et al. 2007b) within 3" from the star and spatial filtering in a $5 \times 5 \lambda / D$ box outside of this radius.

The detection limits where estimated as the azimuthal RMS in $\lambda / D$-width annuli normalized to the reference PSF peak. ADI flux losses where estimated by introducing fake planets into the data cubes at various orientations and separations. The best, worst and median detection limits of the survey are plotted in Fig. 2 (left).

From the observations, 17 previously unknown binaries were identified and rejected during the acquisition. Several hundreds of companion candidates (cc) were identified in the final images and detection maps. Follow-up observations allowed obtaining second epochs for the vast majority of them, confirming no new substellar companions.

\section{First results of the survey}

The survey produced nonetheless two interesting results so far. First, the debris disk around the young G8V star HD 61005 was resolved for the first time as a narrow ring from our NaCo observations (Buenzli et al. 2010). We determined that the ring center is offset by at least $2.75 \pm 0.85$ AU from star. The offset, together with a relatively steep inner rim, could indicate a planetary companion that perturbs the remnant planetesimal belt. From our imaging data we set upper mass limits for companions that exclude any object above the deuterium-burning limit for separations down to 0.3 arcsec. HD 61005 will be a favored target for the forthcoming high-contrast imagers.

The second interesting result is the white dwarf (WD) companion detected around the star HD 8049 (Zurlo et al. 2013). This star was originally estimated as young (<400 Myr), leading to a false estimate of the mass of the companion. Further analysis of the stellar parameters revealed discrepancies between age indicators based on stellar activity and other indicators (lithium abundance, galactic motion), leading to a reevaluation of the age. Moreover, radial velocity trend over almost 30 years was only compatible with a more massive object, leading to the assumption that the companion was in fact a WD, which was later confirmed with VLT/SINFONI $J H K$ spectroscopy. 


\section{Preliminary statistical analysis}

The null result of the survey can be used to infer statistical properties on the population of massive planet at wide orbital separation. For this purpose, we performed Monte-Carlo simulations using the QMESS tool (Bonavita et al. 2013) to generate planets on a regular grid of mass and semi-major axis to obtain probability of detection maps for each of the observed target (Fig. 2, center). Using the formalism previously described in Lafrenière et al. (2007a), and assuming a null-result of the survey, we determine that less than $20 \%$ of stars can harbor planets more massive than $4 \mathrm{M}_{\mathrm{Jup}}$ in the range 55-800 AU (Fig. 2, right). This estimation is compatible with previous studies (e.g. Nielsen \& Close 2010). This analysis is based on the COND evolutionary models (Baraffe et al. 2003).

\section{References}

Baraffe, I., Chabrier, G., Barman, T. S., Allard, F., \& Hauschildt, P. H. 2003, A\&A, 402, 701 Beuzit, J.-L., Feldt, M., Dohlen, K., et al. 2008, in SPIE Conference Series, Vol. 7014 Biller, B. A., Close, L. M., Masciadri, E., et al. 2007, ApJ, 173, 143

Bonavita, M., de Mooij, E. J. W., \& Jayawardhana, R. 2013, PASP, 125, 849

Buenzli, E., Thalmann, C., Vigan, A., et al. 2010, A\&A, 524, L1

Chauvin, G., Lagrange, A.-M., Bonavita, M., et al. 2010, A\&A, 509, A52+

Lafrenière, D., Doyon, R., Marois, C., et al. 2007a, ApJ, 670, 1367

Lafrenière, D., Marois, C., Doyon, R., Nadeau, D., \& Artigau, É. 2007b, ApJ, 660, 770

Marois, C., Lafrenière, D., Doyon, R., Macintosh, B., \& Nadeau, D. 2006, ApJ, 641, 556

Masciadri, E., Mundt, R., Henning, T., Alvarez, C., \& Barrado y Navascués, D. 2005, ApJ, 625, 1004

Nielsen, E. L. \& Close, L. M. 2010, ApJ, 717, 878

Rameau, J., Chauvin, G., Lagrange, A.-M., et al. 2013, A\&A, 553, A60

Vigan, A., Patience, J., Marois, C., et al. 2012, A\&A, 544, A9

Zurlo, A., Vigan, A., Hagelberg, J., et al. 2013, A\&A, 554, A21

\section{Discussion}

L. Close: Can you speak about the fact that HD 8049 looked young in its rotation index, but was in fact old. Is it spin up?

A. Vigan: Yes, our assumption is that it is spin up because of material accreted from the WD progenitor

J. FARIHI: Interpretation of rejuvenation by AGB wind accretion at $a \geqslant 50$ AU should be re-examined. Low mass MS companions to WD, even those in $a<1$ AU orbits, do not exhibit any signs of mass accretion. There are now $\sim 1000$ such WD+MS systems known with no evidence for AGB wind accretion.

S. Metchev: Your effort to exclude stars previously observed at high-contrast is very laudable, but may have introduced unknown biases in sample selection. In particular, no BD discoveries among your 110 stars seems very significant compared to several surveys showing BD frequency of a few percent.

A. Vigan: Our parent sample was 200 stars, from which we excluded 90 previously observed ones, but the statistical analysis will be done on the full sample. In the parent sample, some stars may have already known BD companions (e.g. AB Pic). 